\title{
Chave de famílias de Coleoptera aquáticos (Insecta) do Estado de São Paulo, Brasil
}

\author{
Melissa Ottoboni Segura ${ }^{1,3}$, Francisco Valente-Neto $^{1}$ \& Alaíde Aparecida Fonseca-Gessner ${ }^{1,2}$ \\ ${ }^{1}$ Programa de Pós-Graduação em Ecologia e Recursos Naturais, \\ Universidade Federal de São Carlos - UFSCar, CP 676, CEP 13565-905, São Carlos, SP, Brasil \\ ${ }^{2}$ Departamento de Hidrobiologia, Universidade Federal de São Carlos - UFSCar, \\ CP 676, CEP 13565-905, São Carlos, SP, Brasil \\ ${ }^{3}$ Autor para correspondência: Melissa Ottoboni Segura, e-mail: m_ottoboni@yahoo.com.br
}

\begin{abstract}
SEGURA, M.O., VALENTE-NETO, F. \& FONSECA-GESSNER, A.A. Family level key to aquatic Coleoptera (Insecta) of Sao Paulo State, Brazil. Biota Neotrop. 11(1): http://www.biotaneotropica.org.br/v11n1/en/ abstract?article+bn02711012011.
\end{abstract}

Abstract: Coleoptera is the most species-rich order among the Insecta and although only $4 \%$ of its species are aquatic, it is an abundant group in the freshwater environments. This work aims to expand knowledge of the group primarily in lotic systems. Are proposed identification keys for larvae and adults of 17 families of aquatic Coleoptera known from the state, to assist researchers who are unfamiliar with the identification of this group. All material studied was collected in São Paulo, Brazil as part of the BIOTA/FAPESP Program.

Keywords: aquatic insects, aquatic systems, identification, larvae, adults.

SEGURA, M.O., VALENTE-NETO, F. \& FONSECA-GESSNER, A.A. Chave de famílias de Coleoptera aquáticos (Insecta) do Estado de São Paulo, Brasil. Biota Neotrop. 11(1): http://www.biotaneotropica.org.br/ v11n1/pt/abstract?article+bn02711012011.

Resumo: Coleoptera é a maior ordem de Insecta e apesar de apenas 4\% das espécies serem aquáticas, o grupo apresenta grande abundância em ambientes de água doce. Este trabalho visa ampliar o conhecimento do grupo principalmente em sistemas lóticos. São propostas chaves de identificação para larvas e adultos de 17 famílias de Coleoptera aquáticos conhecidas do Estado, com o objetivo de auxiliar pesquisadores pouco familiarizados com a identificação deste grupo. Todo o material estudado foi coletado no Estado de São Paulo, Brasil, como parte do Programa BIOTA/FAPESP.

Palavras-chave: insetos aquáticos, ambientes aquáticos, identificação, larvas, adultos. 


\section{Introdução}

Coleoptera é a maior ordem de Insecta com cerca de 400 mil espécies em todo o mundo. Apesar de apenas 4\% das espécies serem aquáticas (Jäch \& Balke 2008), o grupo é abundante e diverso entre os macroinvertebrados dos ambientes de água doce. Conforme Crowson (1981), os coleópteros invadiram os ambientes aquáticos cerca de 10 vezes independentemente durante a evolução do grupo. As adaptações morfológicas, fisiológicas e comportamentais encontradas neste grupo são excepcionalmente diversas.

Atualmente são conhecidas cerca de 11 famílias exclusivamente aquáticas nas fases larval e adulta, outras são predominantemente aquáticas como larvas e têm adultos terrestres ou vice versa (Jäch \& Balke 2008). Algumas famílias têm representação aquática esporádica como alguns Chrysomelidae e Curculionidae.

A identificação em família pode ser feita parcialmente com base em caracteres gerais, como tamanho e forma, mas em muitos casos requer uma análise cuidadosa da morfologia externa, particularmente quando ocorrem variações na forma do corpo entre espécies distintas da mesma família. Mas, em geral, não são necessárias observações de características internas para identificar um espécime em família.

Os adultos são caracterizados por um corpo fortemente esclerotizado e possuem como carácter sinapomórfico as asas modificadas em élitros, que servem para proteger as asas membranosas posteriores, tão bem quanto à superfície dorsal dos últimos dois segmentos torácicos e do abdômen. Outras características dos adultos são: a presença de uma gula e antena com 11 artículos.

Em geral, Coleoptera aquáticos ocorrem em uma ampla variedade de hábitats (Larson 1985). São abundantes e diversos em rios, riachos e lagos, particularmente em áreas mais rasas próximas das margens e junto às macrófitas. Também podem ser encontrados em ambientes mais peculiares como poças temporárias, depósitos de água em bromélias ou ocos de troncos de árvores. Algumas famílias são mais comuns em determinados tipos de ambientes, como os Dytiscidae e Hydrophilidae que são mais abundantes em lagos, comparados com os Elmidae comuns em sistemas lóticos, mais frequentemente, em córregos de baixa ordem.

Nos últimos anos tem havido um crescimento de estudos ecológicos em sistemas aquáticos, entre os quais a busca pelo conhecimento de organismos chave para bioavaliações e biomonitoramento da qualidade ambiental. Considerando que esses trabalhos, em geral, são desenvolvidos por não especialistas, a disponibilidade de chaves de identificação podem auxiliar evitando que sejam cometidos erros de identificação.

Neste trabalho, são apresentadas chaves de identificação com a inclusão de 17 famílias, todas coletadas e observadas no Estado de São Paulo, complementando as informações existentes em outras publicações (Mugnai et al. 2010, Benetti et al. 2006) para outras regiões do Brasil.

\section{Material e Métodos}

Os exemplares utilizados para a confecção da chave são provenientes de coletas do projeto de levantamento de Coleoptera aquáticos, parte do Projeto Temático BIOTA/FAPESP: "Levantamento e Biologia de Insecta e Oligochaeta Aquáticos de Sistemas Lóticos do Estado de São Paulo" (proc. 2003/10517-9) e aqueles disponíveis na Coleção do Laboratório de Entomologia Aquáticos da Universidade Federal de São Carlos (UFSCar). O material analisado foi coletado no período de 2005 a 2010, utilizando diferentes técnicas de amostragem em 15 Bacias Hidrográficas no Estado de São Paulo: Araraquara (21 $\left.{ }^{\circ} 50^{\prime} \mathrm{S} / 48^{\circ} 08^{\prime} \mathrm{W}\right)$; Atibaia (23 $\left.09^{\prime} \mathrm{S} / 46^{\circ} 30^{\prime} \mathrm{W}\right)$; Botucatu (22 $48^{\prime}$ S $\left./ 48^{\circ} 24^{\prime} \mathrm{W}\right)$; Campos do Jordão, Parque Estadual

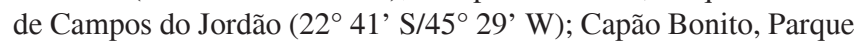
Estadual de Intervales (24 16' S/48 $27^{\prime}$ W); Gália, Estação Ecológica de Caetetus (22 ${ }^{\circ} 23^{\prime}$ S $/ 49^{\circ} 41^{\prime}$ W); Luis Antônio, Estação Ecológica Jataí ( $21^{\circ} 36^{\prime}$ S $/ 47^{\circ} 48^{\prime} \mathrm{W}$ ); Pedregulho, Parque Estadual das Furnas do Bom Jesus $\left(20^{\circ} 13^{\prime}\right.$ S $\left./ 47^{\circ} 27^{\prime} \mathrm{W}\right)$; Santa Rita do Passa Quatro, Parque Estadual de Vassununga ( $21^{\circ} 38^{\prime}$ S $/ 47^{\circ} 37^{\prime}$ W); São Carlos (22 $02^{\prime}$ S $/ 47^{\circ} 46^{\prime}$ W); São José do Rio Preto (20³3' S/49 ${ }^{\circ} 14^{\prime}$ W); São Luiz do Paraitinga, Parque Estadual da Serra do Mar - Núcleo

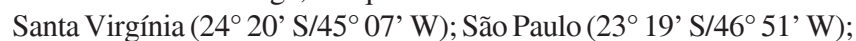
Teodoro Sampaio, Parque Estadual do Morro do Diabo (22 $36^{\circ}$ S/ $\left.52^{\circ} 18^{\prime} \mathrm{W}\right)$; Ubatuba, Parque Estadual da Serra do Mar - Núcleo Picinguaba $\left(23^{\circ} 22^{\prime} \mathrm{S} / 44^{\circ} 46^{\prime} \mathrm{W}\right)$.

Neste trabalho são incluídas somente as famílias que possuem espécies com representantes aquáticos em pelo menos um estágio de vida (Costa et al. 1988, Lopretto \& Tell 1995, Jäch \& Balke 2008, Archangelsky et al. 2009). A terminologia utilizada está de acordo com Costa et al. 1988 para larvas e Lopretto \& Tell 1995 para adultos.

As imagens foram realizadas com uma câmera Leica DFC 280 acoplada a um estereomicroscópio Leica MZ95. Posteriormente, as imagens foram tratadas para correções de contraste, brilho, imperfeições e padronização de tamanho através do programa Adobe Photoshop CS3.

\section{Resultados e Discussão}

A chave de identificação de Coleoptera aquáticos para o Estado de São Paulo inclui 17 famílias, entre as quais algumas são exclusivamente aquáticas na maior parte do seu ciclo vital, como: Dytiscidae, Noteridae, Gyrinidae, Hydrophilidae, Haliplidae, Elmidae, Hydroscaphidae, Hydraenidae, Dryopidae, Lutrochidae, Torrindincolidae e Hydrophilidae. Outras famílias incluem exemplares que vivem nas margens dos corpos de água, os adultos são terrestres e as larvas aquáticas, como Psephenidae, Ptilodactylidae e Scirtidae; e ainda famílias tipicamente terrestres com algumas espécies aquáticas, como Curculionidae, Lampyridae e Staphylinidae. No presente trabalho entre as famílias exclusivamente aquáticas registradas para o Brasil apenas Haliplidae não foi registrada (Benetti et al. 2003), talvez por não ter sido realizadas coletas em ambientes onde estes coleópteros ocorrem. Os Coleoptera aquáticos ocupam uma grande variedade de ambientes. As famílias pertencentes à subordem Adephaga, (Dytiscidae, Gyrinidae e Noteridae), além de Hydrophilidae (Polyphaga) são abundantes e frequentes em ambientes lênticos, mas podem ser encontrados em ambientes lóticos, principalmente em áreas de remanso. Em córregos são típicas as famílias incluídas no grupo "Riffle Beetles", Elmidae, Psephenidae, Dryopidae e Lutrochidae, encontradas em águas correntes e bem oxigenadas (Brown 1987). A família Torridincolidae (Myxophaga) ocorre exclusivamente em ambientes higropétricos, paredões rochosos por onde escorre uma delgada lâmina de água. As demais famílias observadas neste trabalho são comuns em ambientes lóticos e lênticos. Ressalta-se a dificuldade na separação de larvas de Elmidae e Lutrochidae que é feito em parte com base na presença e número de brânquias anais, uma vez que estas em geral ficam retraídas em exemplares fixados. 


\section{Larvas}

1. Pernas.

1'. Pernas vestigiais ou ausentes.

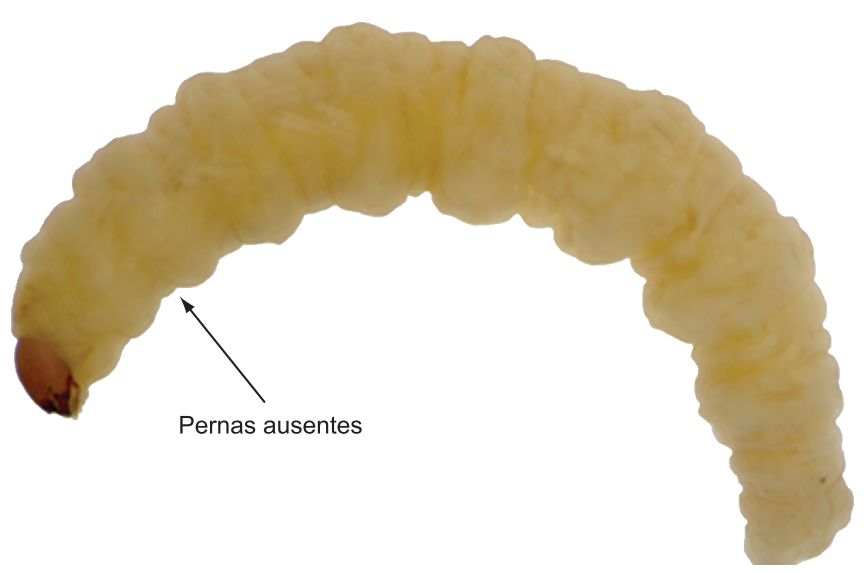

Figura 1. Curculionidae, larva, vista lateral.

Figure 1. Curculionidae, larvae, lateral view.

2. Pernas com 6 segmentos, incluindo um par de garras

.3 (Figura 2)

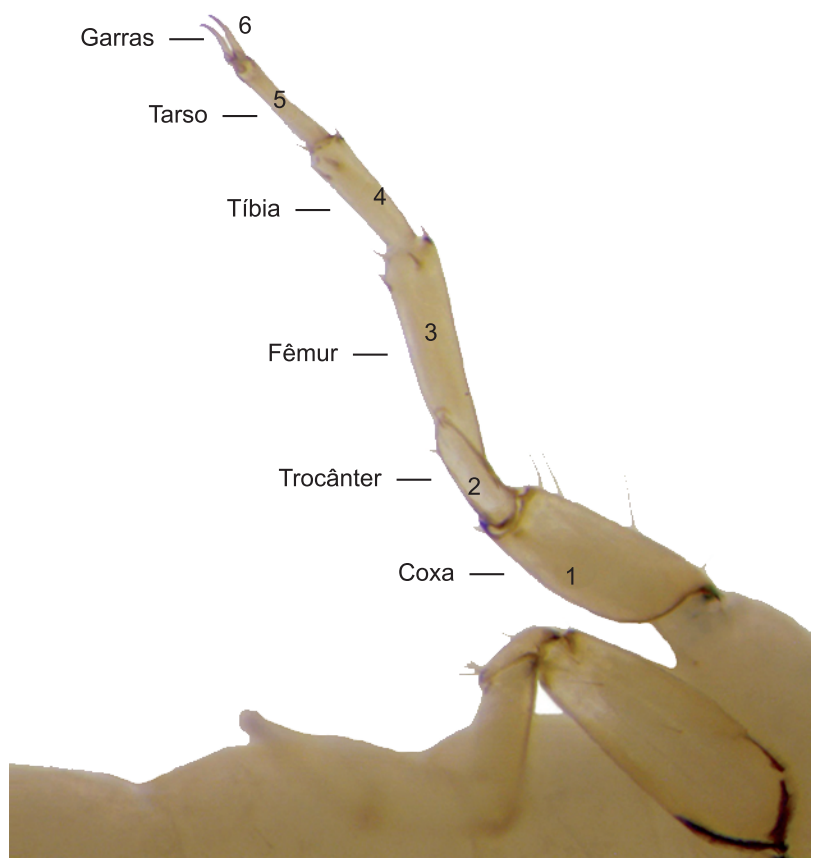

Figura 2. Gyrinidae, larva,vista látero-ventral das pernas.

Figure 2. Gyrinidae, larvae, latero- ventral view of the legs. 


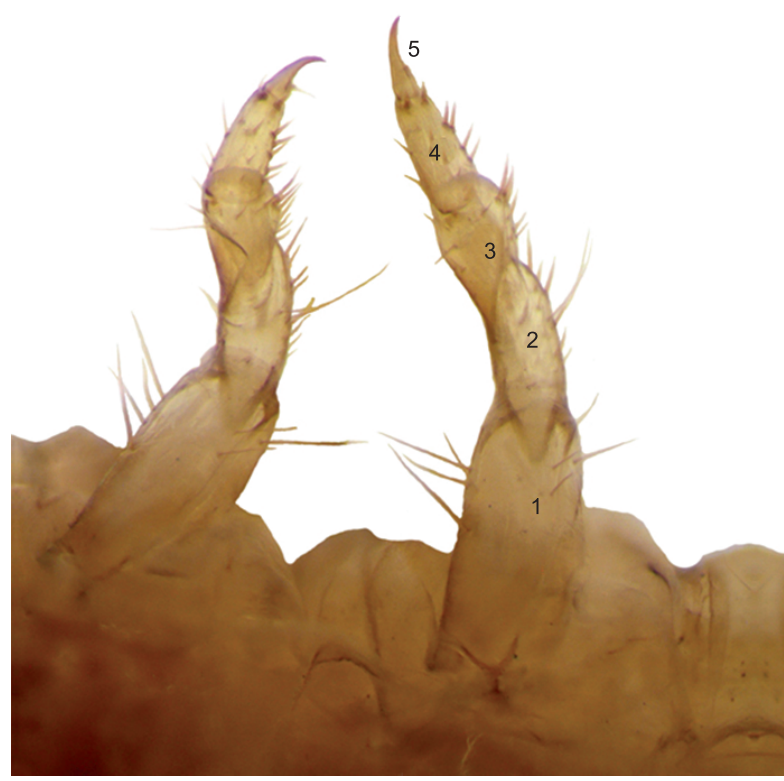

Figura 3. Ptilodactylidae, larva,vista látero-ventral das pernas.

Figure 3. Ptilodactylidae, larvae, latero-ventral view of the legs.

3. Brânquias laterais franjadas presentes em todos os segmentos abdominais, extremidade do último segmento abdominal com quatro ganchos Gyrinidae (Figuras 4 e 5)

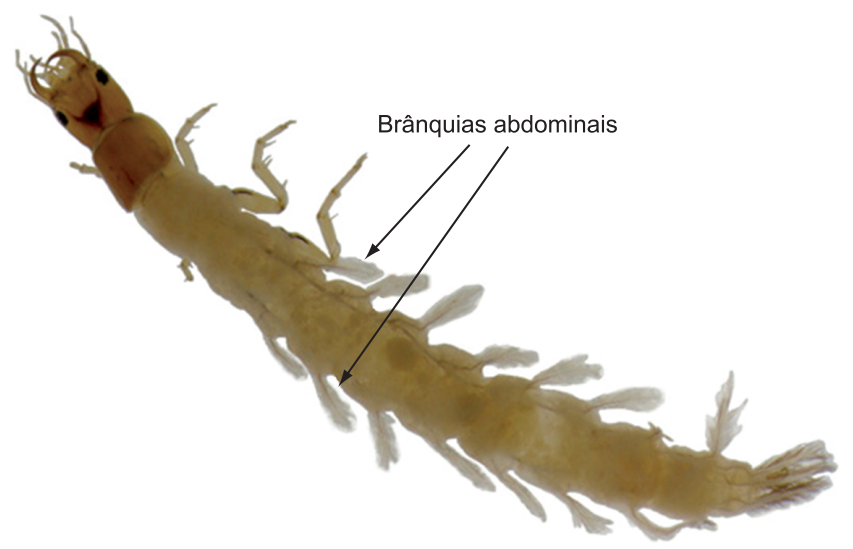

Figura 4. Gyrinidae, larva, vista dorsal.

Figure 4. Gyrinidae, larvae, dorsal view.

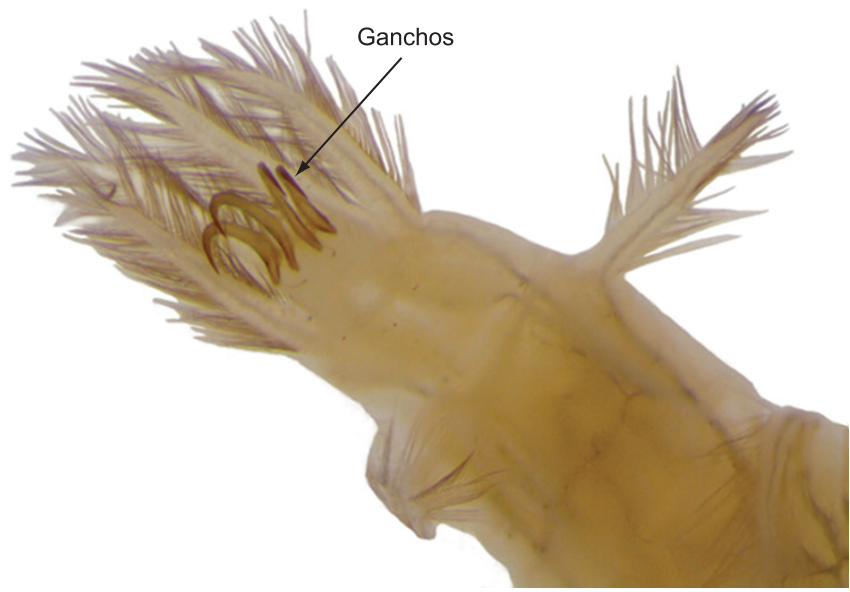

Figura 5. Gyrinidae, larva, vista ventral da região posterior.

Figure 5. Gyrinidae, larvae, ventral view of the posterior region. 
3'. Segmentos abdominais com ou sem brânquias laterais, último segmento abdominal sem ganchos......

4. Corpo convexo; cabeça sem constrição posterior; mandíbulas curtas, largas na base e estreitas no ápice... Noteridae (Figuras 6 e 7)

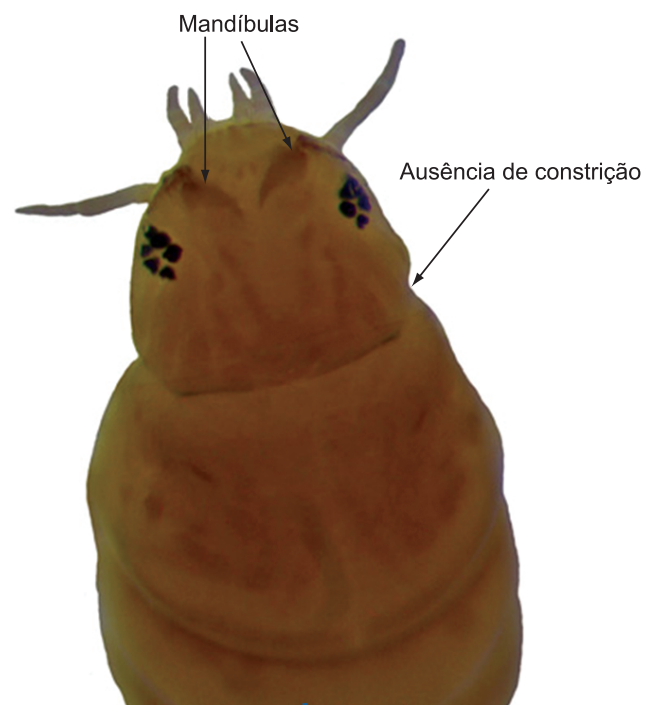

Figura 6. Noteridae, larva, vista dorsal da região anterior.

Figure 6. Noteridae, larvae, dorsal view of the anterior region.

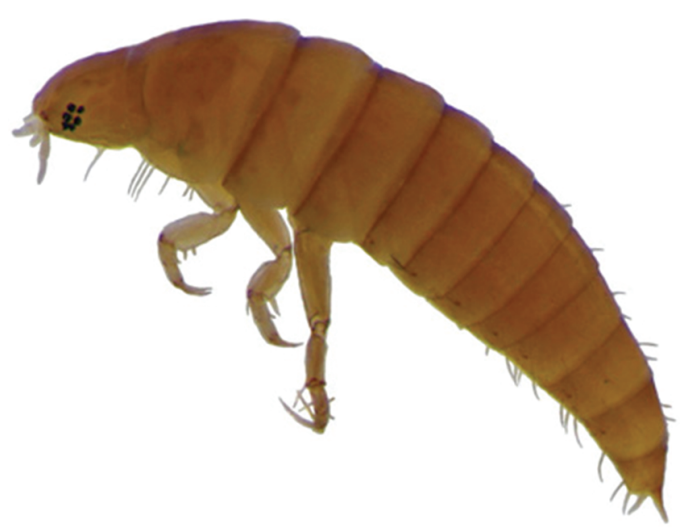

Figura 7. Noteridae, larva, vista lateral.

Figure 7. Noteridae, larvae, lateral view.

4'. Corpo alongado; cabeça geralmente com constrição posterior; mandíbulas em geral longas e delgadas, falciforme Dytiscidae (Figuras 8 e 9)

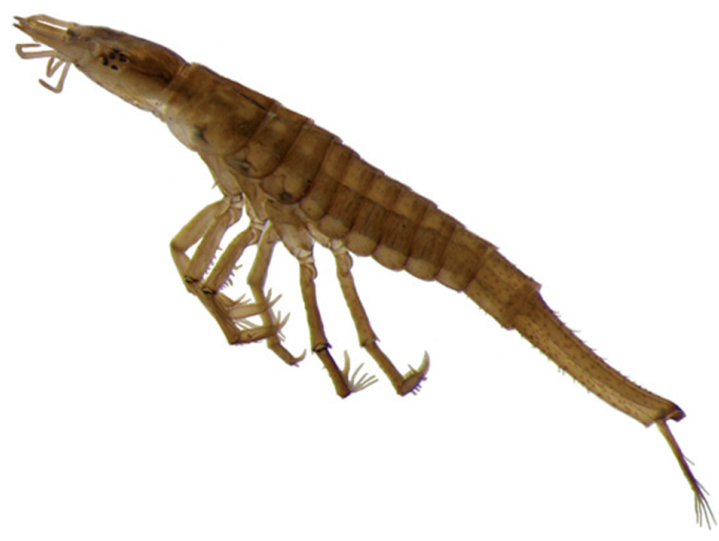

Figura 8. Dytiscidae, larva, vista lateral.

Figure 8. Dytiscidae, larvae, lateral view. 


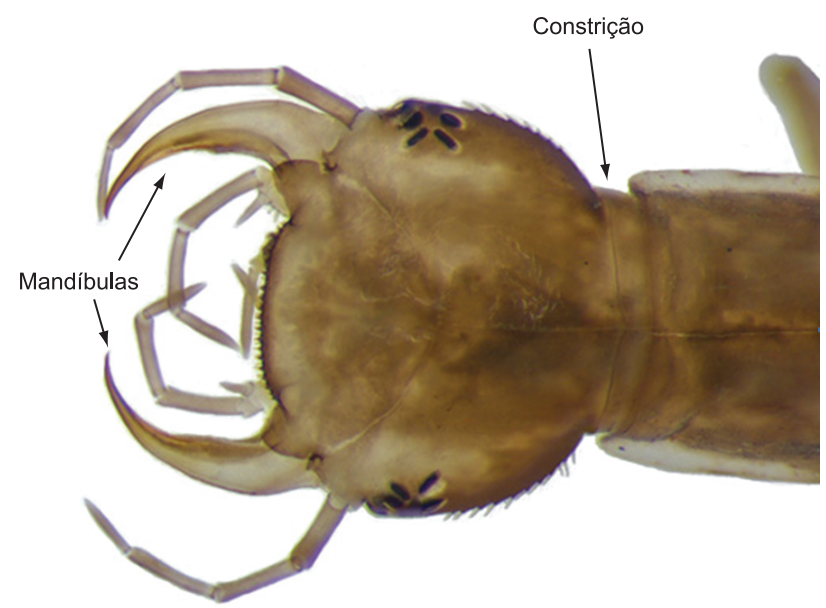

Figura 9. Dytiscidae, larva, vista dorsal da cabeça e pronoto.

Figure 9. Dytiscidae, larvae, dorsal view of the head and pronotum.

5. Labro e clípeo fundidos

6 (Figura 10)

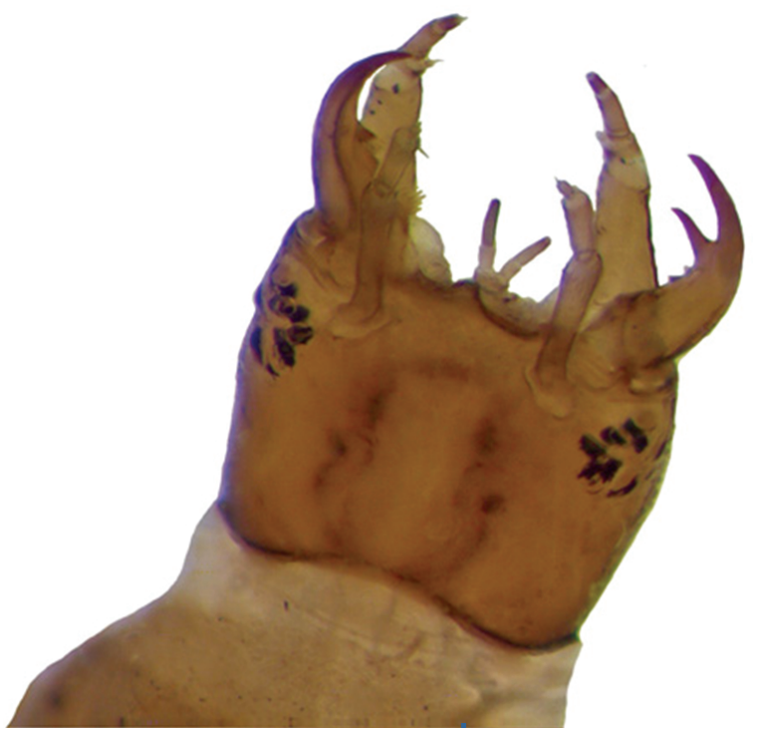

Figura 10. Hydrophilidae, larva, vista dorsal da cabeça e pronoto.

Figure 10. Hydrophilidae, larvae, dorsal view of the head and pronotum.

5'. Labro e clípeo separados por uma sutura evidente.

7 (Figura 11)

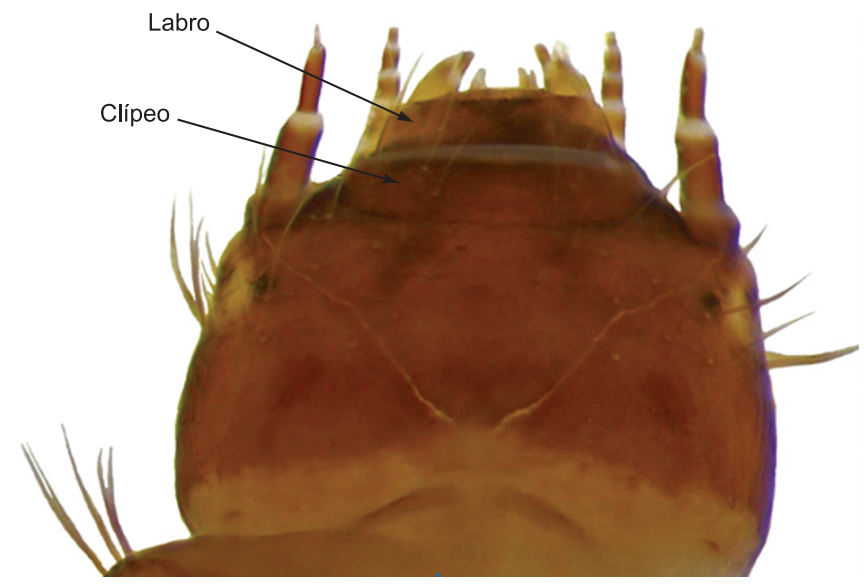

Figura 11. Ptilodactylidae, larva, vista dorsal da cabeça.

Figure 11. Ptilodactylidae, larvae, dorsal view of the head. 
6. Corpo oval alongado, deprimido; cabeça total ou parcialmente retraída no protórax; tergitos bem esclerotinizados e expandidos lateralmente formando projeções e voltadas para trás. .Lampyridae (Figuras 12 e 13)

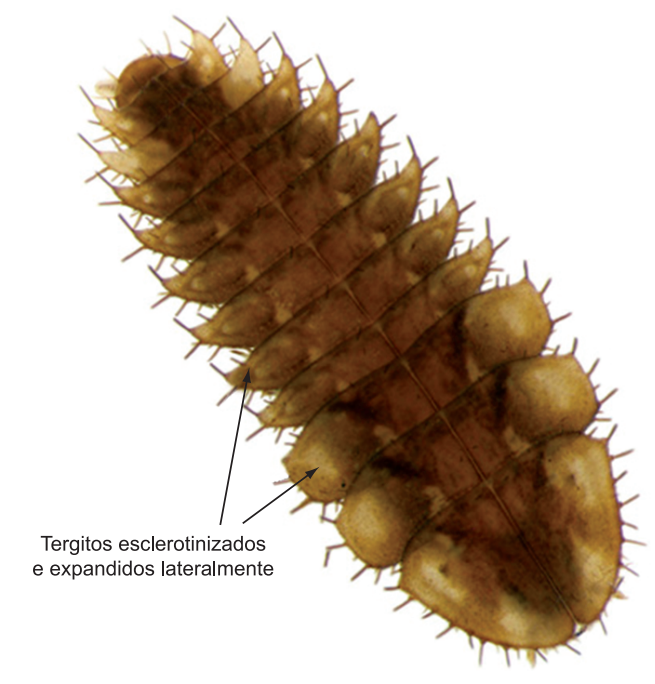

Figura 12. Lampyridae, larva, vista dorsal.

Figure 12. Lampyridae, larvae, dorsal view.

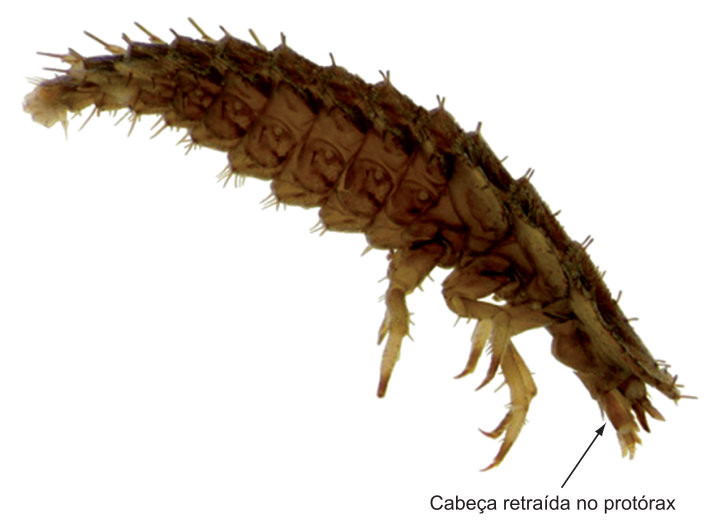

Figura 13. Lampyridae, larva, vista lateral.

Figure 13. Lampyridae, larvae, lateral view.

6'. Corpo alongado; cabeça visível dorsalmente; corpo pouco esclerotinizado, com exceção da cabeça e de placas esclerotinizadas, geralmente existentes nos tergitos torácicos e $8^{\circ}$ tergito abdominal...... Hydrophilidae (Figuras 14 e 15)

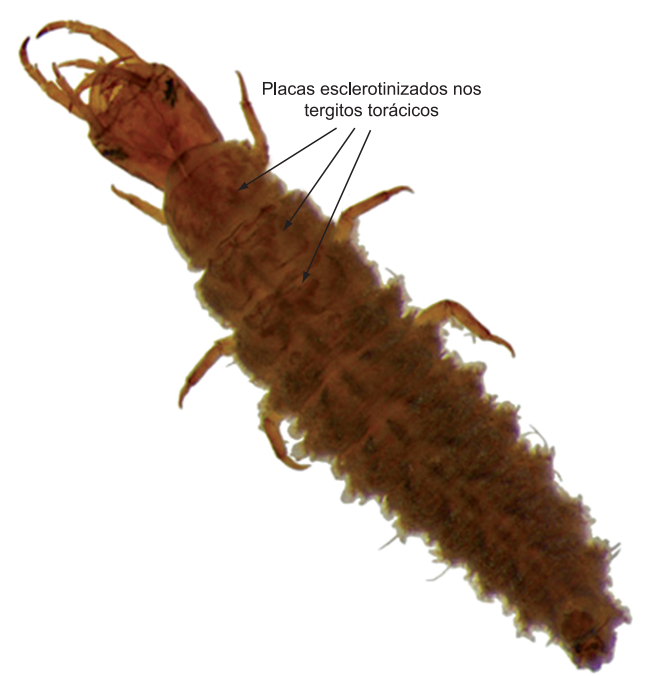

Figura 14. Hydrophilidae, larva, vista dorsal.

Figure 14. Hydrophilidae, larvae, dorsal view. 


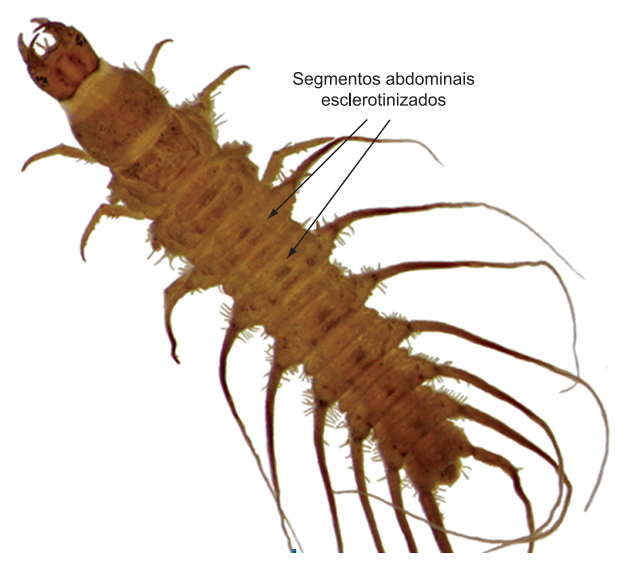

Figura 15. Hydrophilidae, larva, vista dorsal.

Figure 15. Hydrophilidae, larvae, dorsal view.

7. Antenas com dois segmentos basais grandes e um flagelo pluriarticulado; mais longas que o comprimento da cabeça mais o tórax........ Scirtidae (Figura 16)

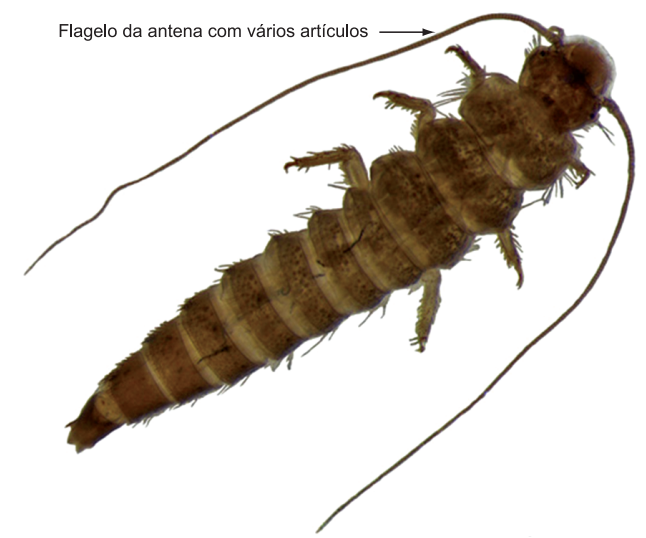

Figura 16. Scirtidae, larva, vista dorsal.

Figure 16. Scirtidae, larvae, dorsal view.

7'. Antenas curtas menores que o comprimento da cabeça mais o tórax, com 3 ou menos segmentos

8. Tamanho pequeno ( $\leq 2 \mathrm{~mm}$ ); antenas com dois segmentos; brânquias abdominais laterais digitiformes presentes nos segmentos 1-8 (em ambientes higropétricos)...... Torridincolidae (Figuras 17 e 18)

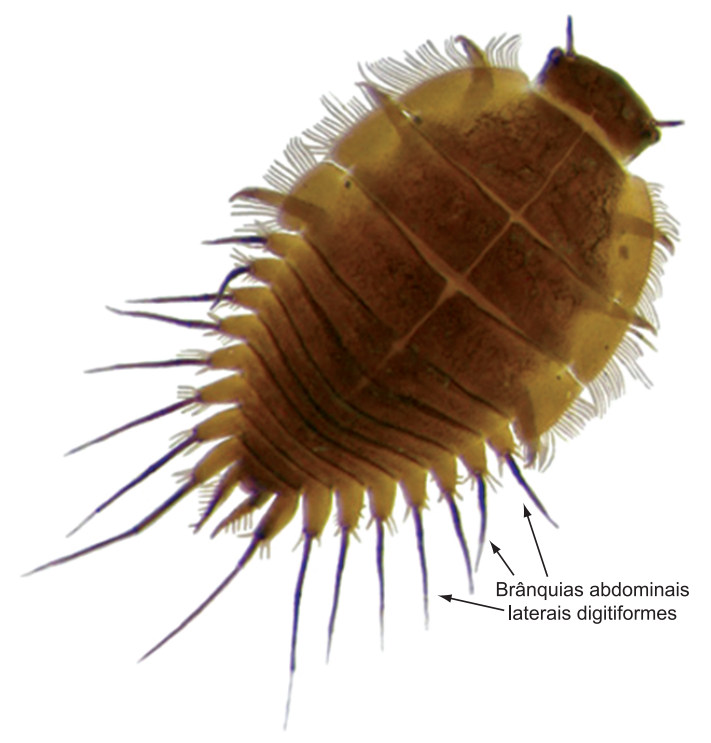

Figura 17. Torridincolidae, larva, vista dorsal.

Figure 17. Torridincolidae, larvae, dorsal view. 


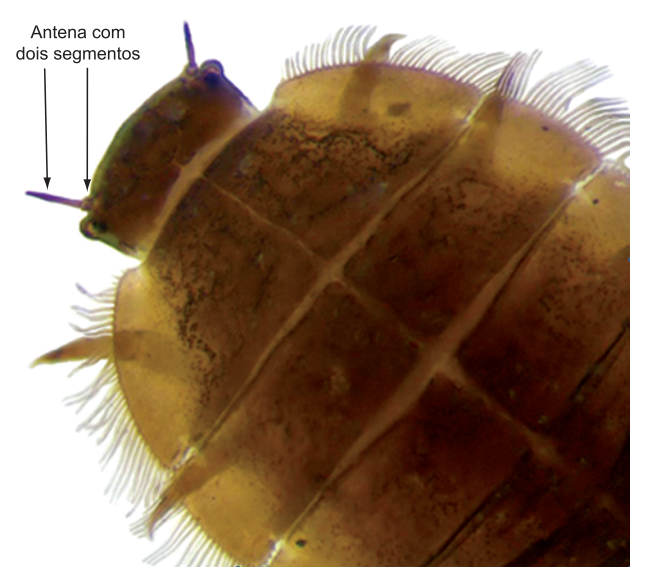

Figura 18. Torridincolidae, larva, vista dorsal da região anterior.

Figure 18. Torridincolidae, larvae, dorsal view of the anterior region.

8'. Tamanho maior que $2 \mathrm{~mm}$; brânquias abdominais, quando presentes, com um par de tufos brânquiais ventrais

9. Corpo muito deprimido, em geral oval e disciforme com segmentos torácicos e abdominais expandidos; cabeça completamente encoberta dorsalmente pelas expansões tergais; região ventral do abdômen com tufos de brânquias (Psepheninae e Eubrianacinae) ou com $9^{\circ}$ esternito formando opérculo, que encerra tufos de brânquias anais (Eubriinae e Psephenoidinae) Psephenidae (Figuras 19, 20, 21 e 22)

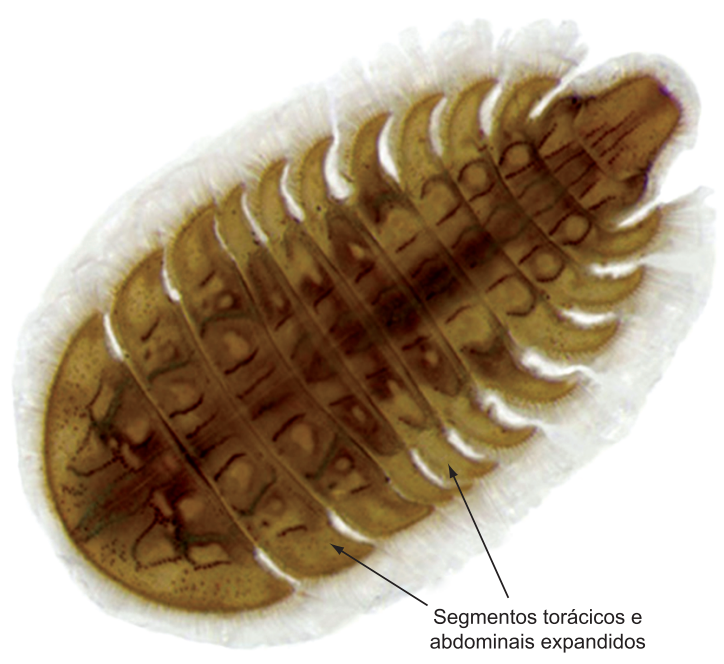

Figura 19. Psephenidae (Eubriinae), larva, vista dorsal. Nota: quando o organismo é fixado as brânquias podem ficar retraídas no interior do último segmento abdominal.

Figure 19. Psephenidae (Eubriinae), larvae, dorsal view. Note: The gills may be withdrawn within the last abdominal segment in preserved specimens.

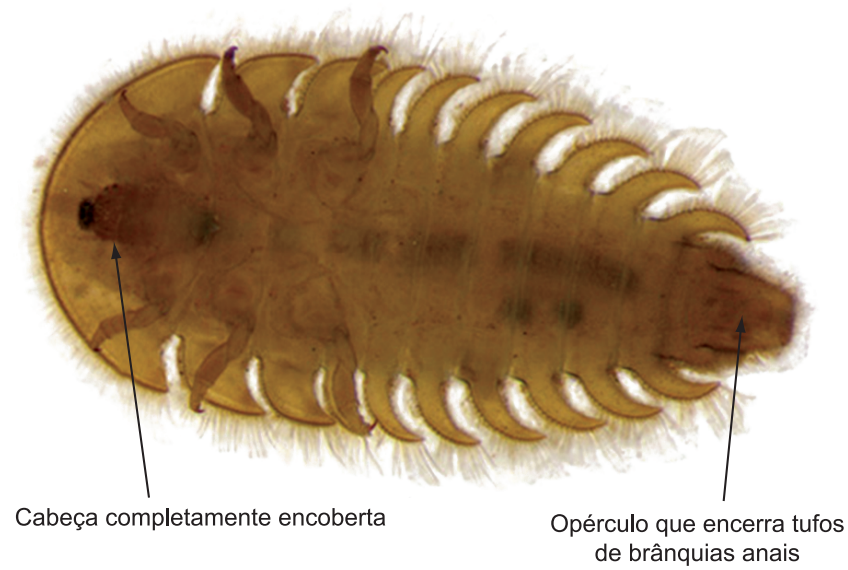

Figura 20. Psephenidae (Eubriinae), larva, vista ventral.

Figure 20. Psephenidae (Eubriinae), larvae, ventral view. 


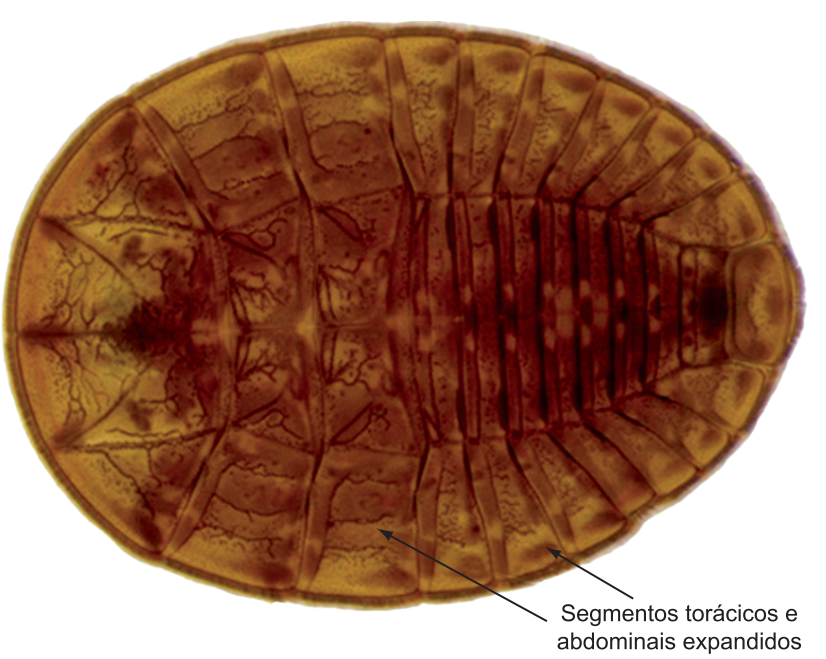

Figura 21. Psephenidae (Psepheninae), larva, vista dorsal.

Figure 21. Psephenidae (Psepheninae), larva, dorsal view.

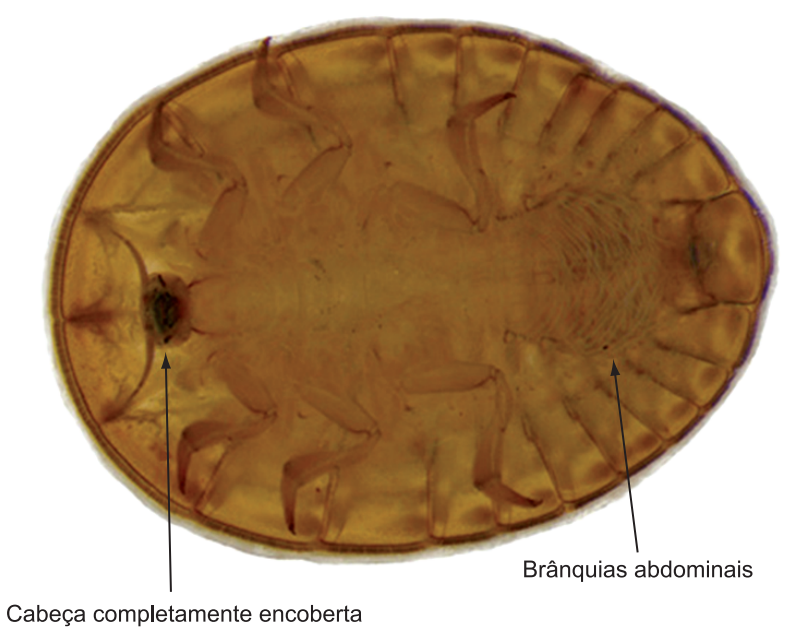

Figura 22. Psephenidae (Psepheninae), larva, vista ventral.

Figure 22. Psephenidae (Psepheninae), larvae, ventral view.

9'. Corpo com formato distinto, sem brânquias abdominais

10. Último segmento abdominal com opérculo ventral que cobre a região anal

10'. Último segmento sem opérculo ventral, com um par de lobos anais, cada um desses provido com vários ganchos Ptilodactylidae (Figuras 23 e 24)

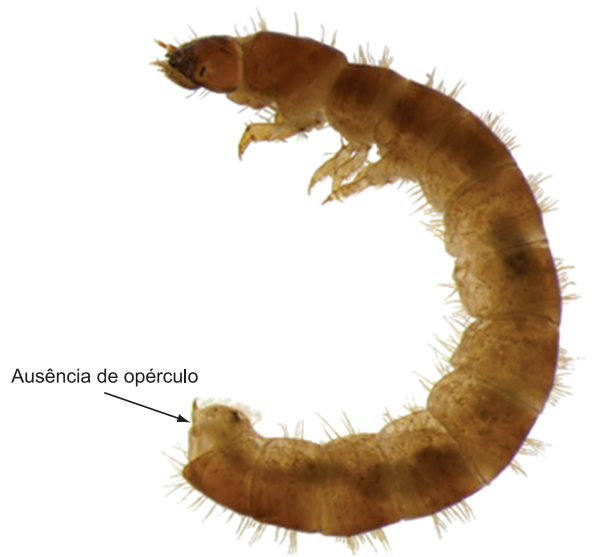

Figura 23. Ptilodactylidae, larva, vista látero-ventral.

Figure 23. Ptilodactylidae, larvae, latero-ventral view. 


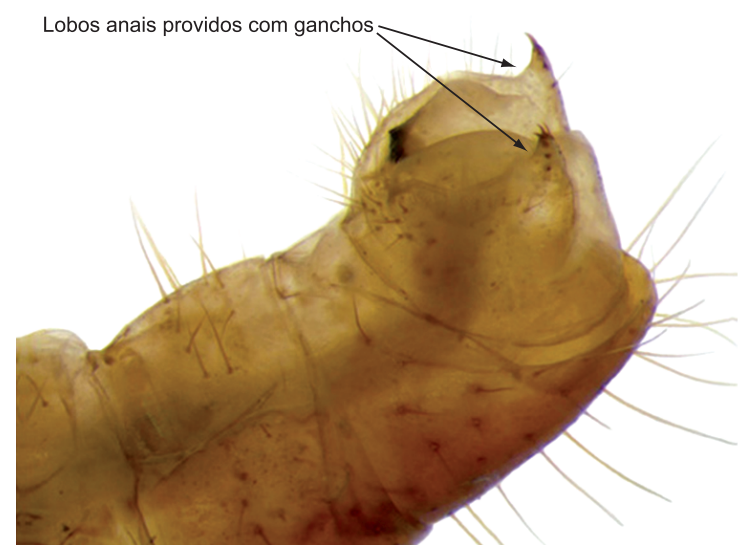

Figura 24. Ptilodactylidae, larva, vista lateral da região posterior.

Figure 24. Ptilodactylidae, larvae, lateral view of the posterior region.

11. Tufos de brânquias anais presentes

11'. Tufos de brânquias anais ausentes; corpo cilíndrico; esternitos abdominais reduzidos ou ausentes, de tal modo que os tergitos são anulares.

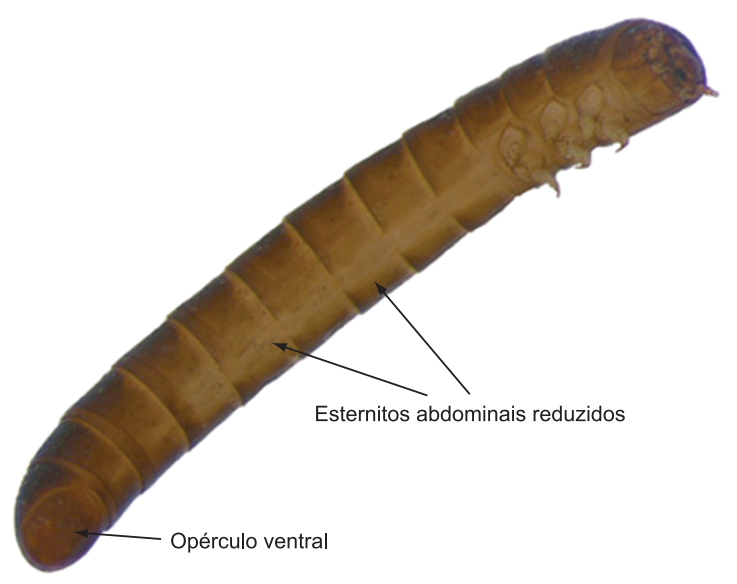

Figura 25. Dryopidae, larva, vista ventral.

Figure 25. Dryopidae, larvae, ventral view.

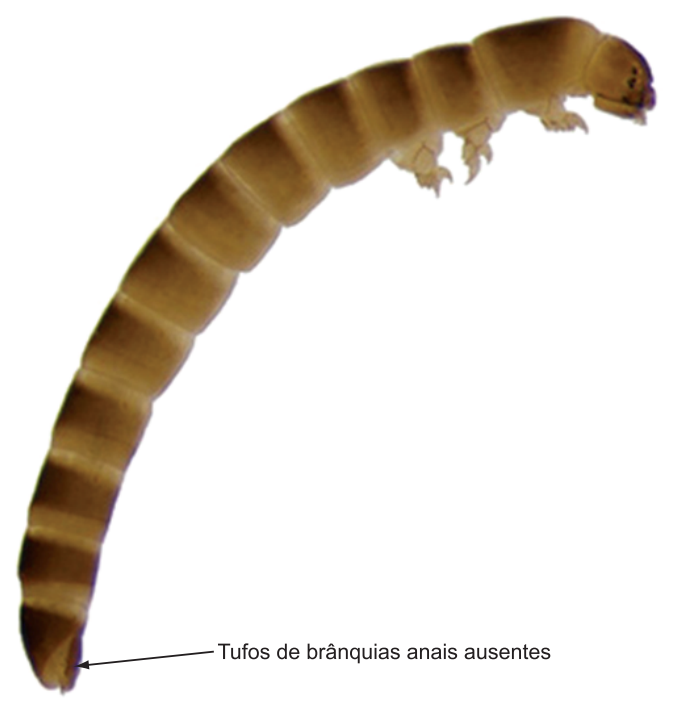

Figura 26. Dryopidae, larva, vista lateral.

Figure 26. Dryopidae, larvae, lateral view. 
12. Pleuritos presentes pelo menos nos quatro primeiros segmentos abdominais; último tergito abdominal sempre modificado, variando de forma, com três tufos de brânquias. .Elmidae (Figuras 27 e 28)

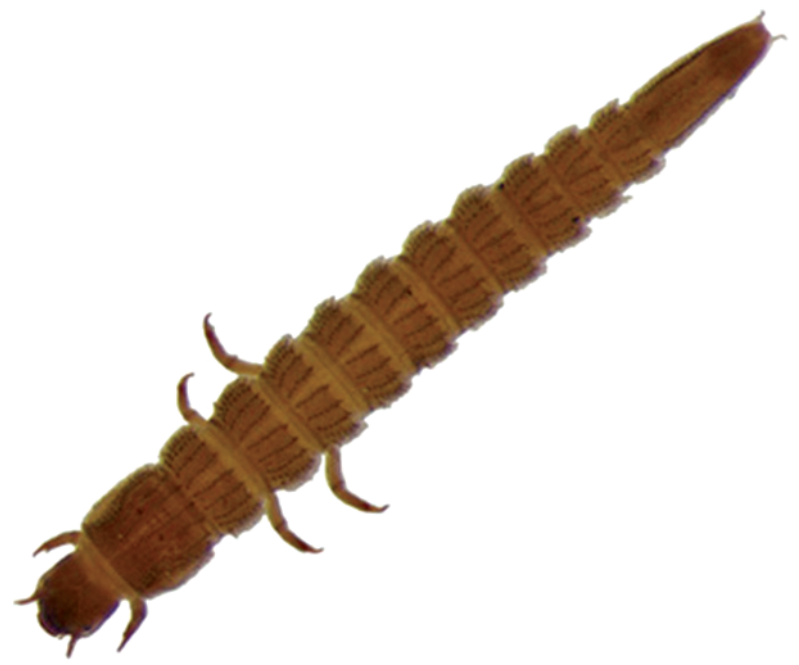

Figura 27. Elmidae, larva, vista dorsal. Nota: quando o organismo é fixado as brânquias podem ficar retraídas no interior do último segmento abdominal. Figure 27. Elmidae, larvae, dorsal view. Note: The gills may be withdrawn within the last abdominal segment in preserved specimens.

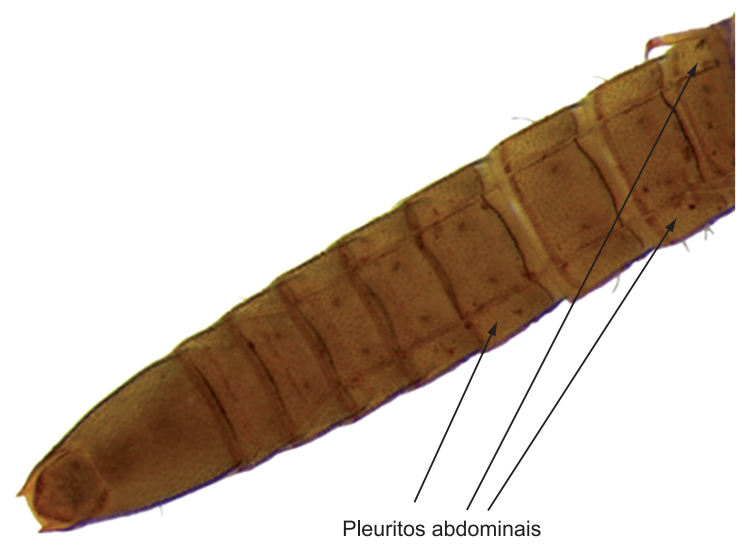

Figura 28. Elmidae, larva, vista ventral da região posterior.

Figure 28. Elmidae, larvae, ventral view of the posterior region.

12'. Pleuritos presentes apenas nos três primeiros segmentos abdominais; ápice do último tergito abdominal arredondado, com um tufo de brânquias. .Lutrochidae (Figuras 29 e 30)

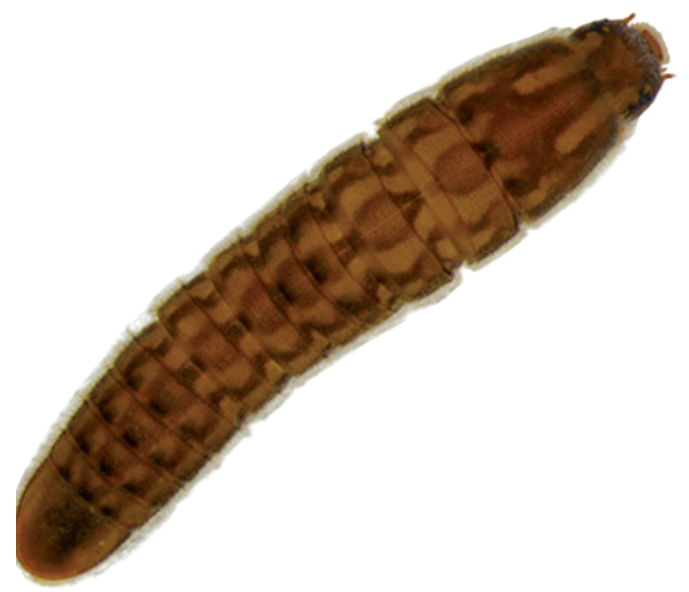

Figura 29. Lutrochidae, larva, vista dorsal. Nota: quando o organismo é fixado as brânquias podem ficar retraídas no interior do último segmento abdominal. Figure 29. Lutrochidae, larvae, dorsal view. Note: The gills may be withdrawn within the last abdominal segment in preserved specimens. 


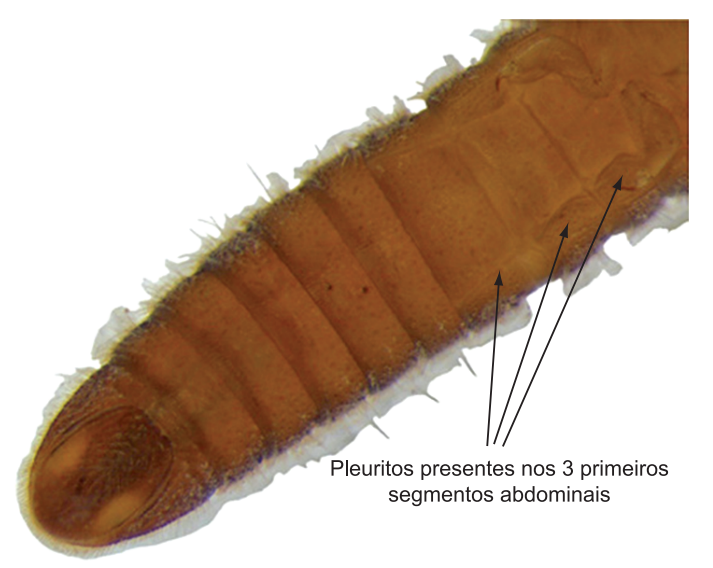

Figura 30. Lutrochidae, larva, vista ventral da região posterior.

Figure 30. Lutrochidae, ventral view of the posterior region.

\section{Adultos}

1. Primeiro ventrito dividido pelas coxas

Subordem Adephaga 2 (Figura 31)

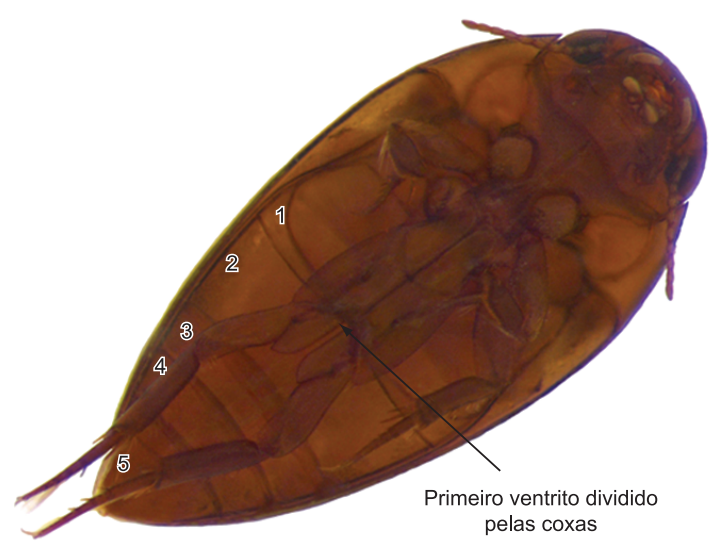

Figura 31. Noteridae, adulto, vista ventral.

Figure 31. Noteridae, adult, ventral view.

1'. Primeiro ventrito não dividido pelas coxas .Subordens Polyphaga/Myxophaga 4 (Figura 32)

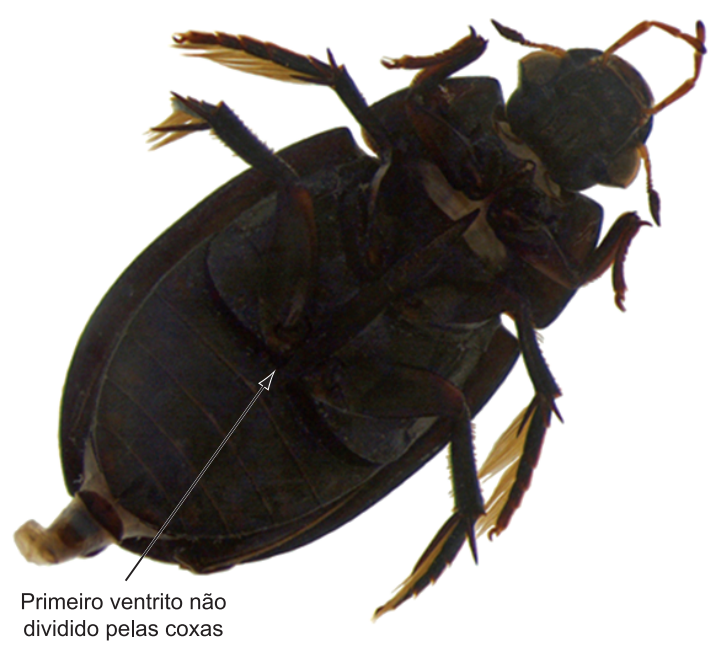

Figura 32. Hydrophilidae, adulto, vista ventral.

Figure 32. Hydrophilidae, adult, ventral view. 
2. Olhos compostos divididos (um dorsal e outro ventral); pernas medianas e posteriores curtas e alargadas adaptadas a natação como aletas. . Gyrinidae (Figuras 33 e 34)

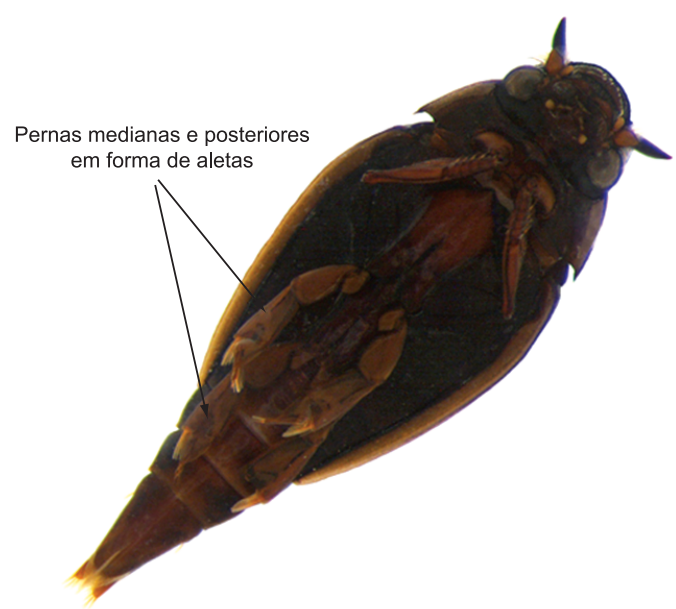

Figura 33. Gyrinidae, adulto, vista ventral.

Figure 33. Gyrinidae, adult, ventral view.

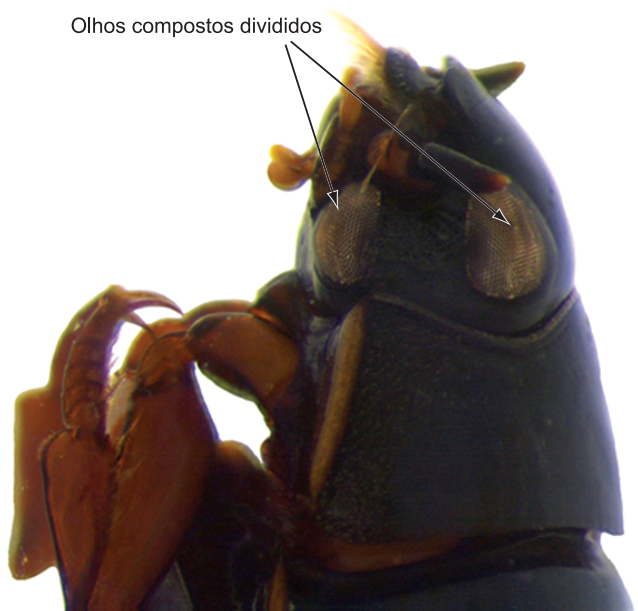

Figura 34. Gyrinidade, adulto, vista lateral da cabeça e pronoto.

Figure 34. Gyrinidae, adult, lateral view of the head and pronotum.

2'. Olhos compostos não divididos; pernas medianas e posteriores nunca como aletas .....

3. Apófise metaesternal alargada, em forma de placa.

Noteridae (Figura 35)

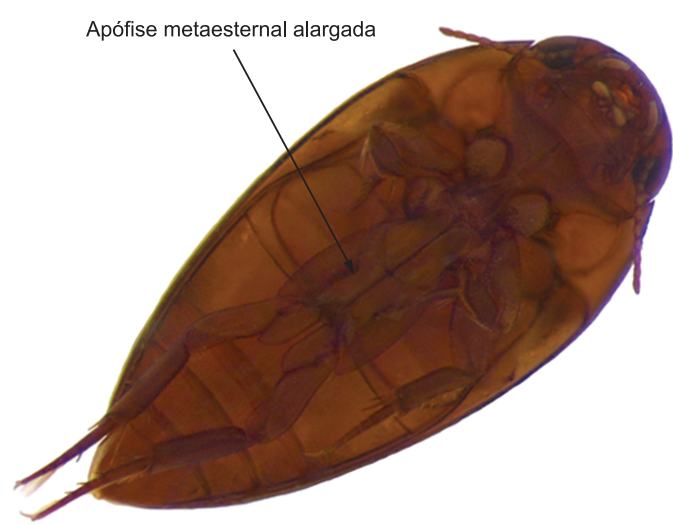

Figura 35. Noteridae, adulto, vista ventral.

Figure 35. Noteridae, adult, ventral view. 


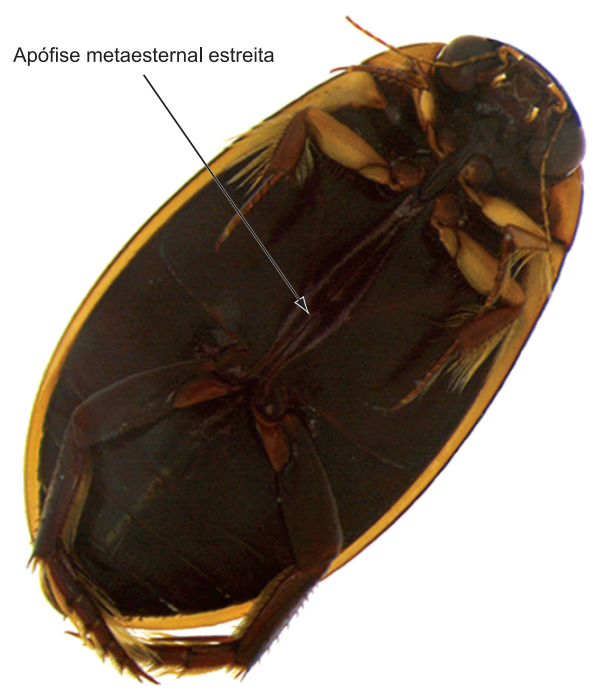

Figura 36. Dytiscidae, adulto, vista ventral.

Figure 36. Dytiscidae, adult, ventral view.

4. Élitros curtos, pelo menos dois tergitos abdominais visíveis

4'. Élitros tão longos como o abdômen, podem deixar o último tergito abdominal visível

5. Antenas com 10-11 antenômeros; pelo menos seis tergitos abdominais visíveis.

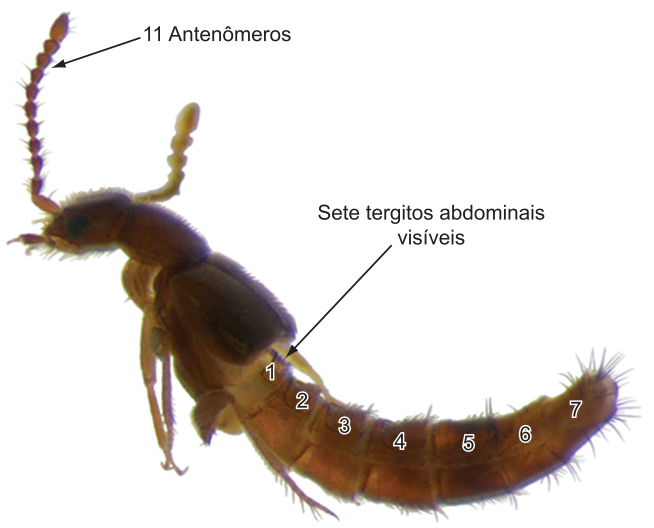

Figura 37. Staphylinidae, adulto, vista lateral.

Figure 37. Staphylinidae, adult, lateral view.

5'. Somente 3-5 tergitos abdominais visíveis; antenas de 8 ou menos antenômeros

Hydroscaphidae (Figura 38)

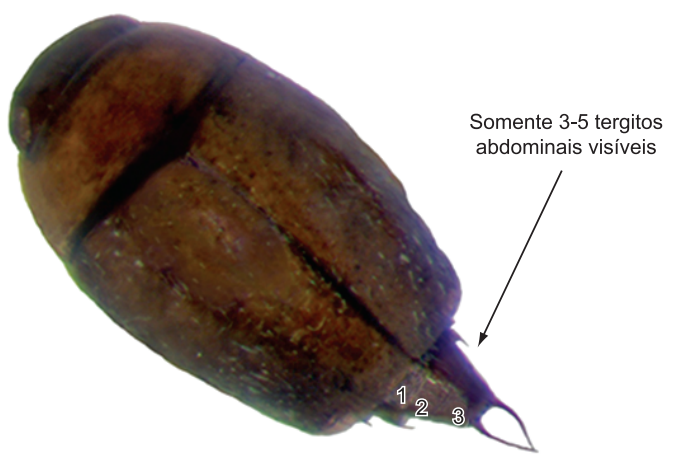

Figura 38. Hydroscaphidae, adulto, vista dorsal.

Figure 38. Hydroscaphidae, adult, dorsal view.

6. Antenas curtas, o último robusto e tão longo quanto a soma dos antenômeros 2 a 6 ; menores que 2 mm (em ambientes higropétricos).. Torridincolidae (Figura 39) 


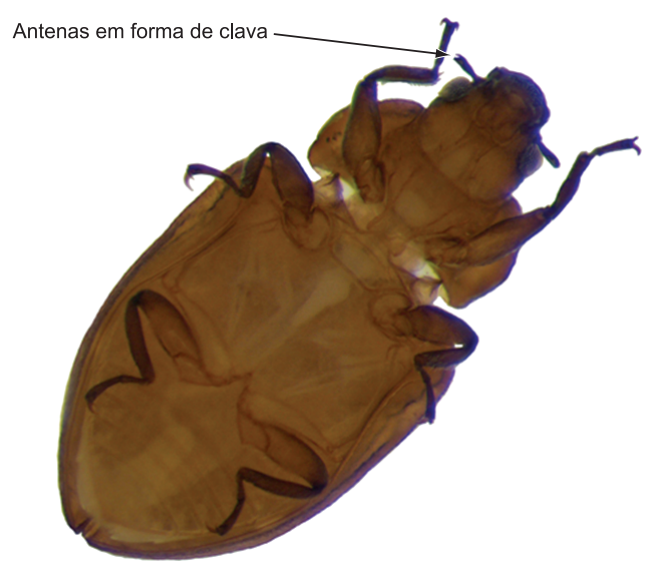

Figura 39. Torridincolidae, adulto, vista ventral.

Figure 39. Torridincolidae, adult, ventral view.

6'. Antenas diferentes da anterior; tamanho variado, em geral, maior que $2 \mathrm{~mm}$.

7. Cabeça modificada em um rostro mais longo do que largo, com as antenas inseridas na região mediana deste

Curculionidae (Figuras 40 e 41)

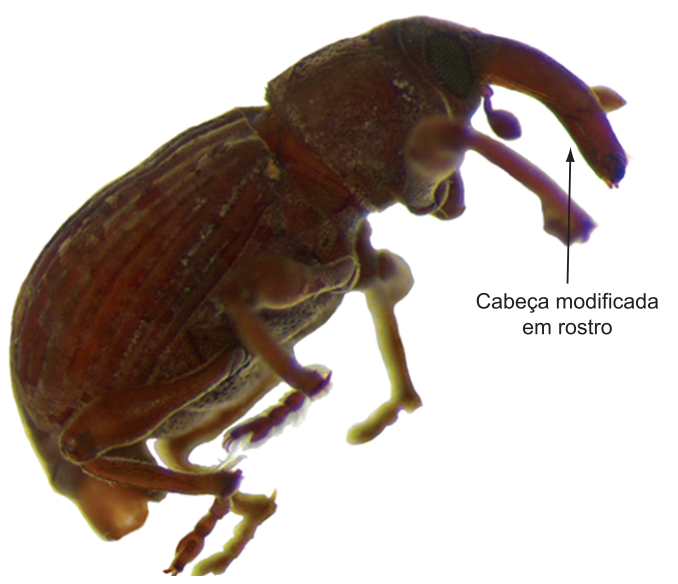

Figura 40. Curculionidae, adulto, vista lateral.

Figure 40. Curculionidae, adult, lateral lateral.

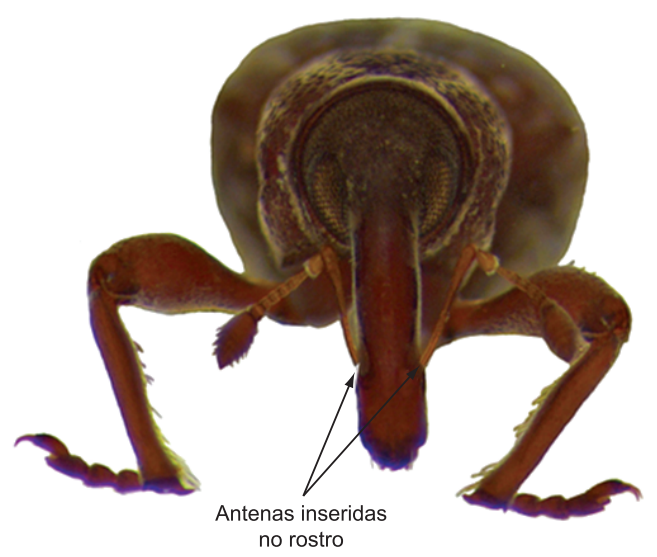

Figura 41. Curculionidae, adulto, vista frontal da cabeça.

Figure 41. Curculionidae, adult, frontal view of the head.

7'. Cabeça não modificada em um rostro.

8. Coxas medianas muito separadas, coxas posteriores próximas ou contíguas; pernas posteriores retrateis encaixadas em um sulco no primeiro ventrito 


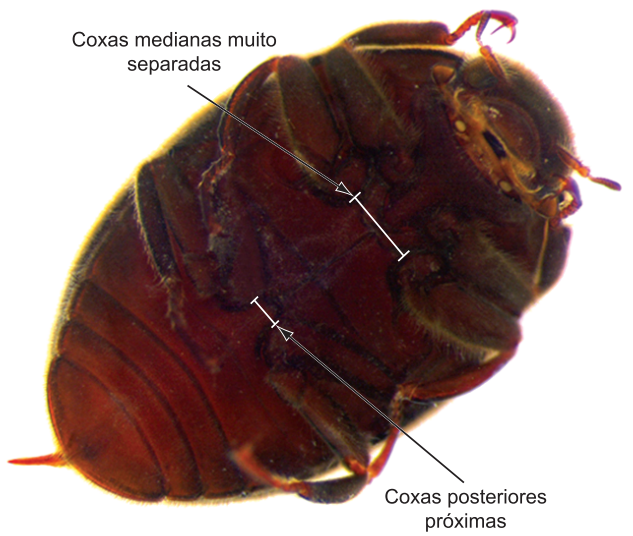

Figura 42. Lutrochidae, adulto, vista ventral.

Figure 42. Lutrochidae, adult, ventral view.

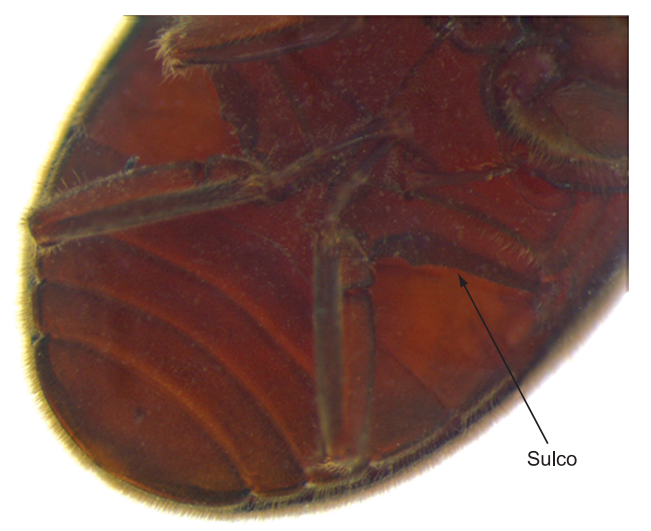

Figura 43. Lutrochidae, adulto, vista ventral da região posterior.

Figure 43. Lutrochidae, adult, ventral view of the posterior region.

8'. Coxas medianas pouco separadas, porém se separadas, as coxas posteriores são tão ou mais separadas que as medianas; pernas posteriores não retráteis .9 (Figura 44)

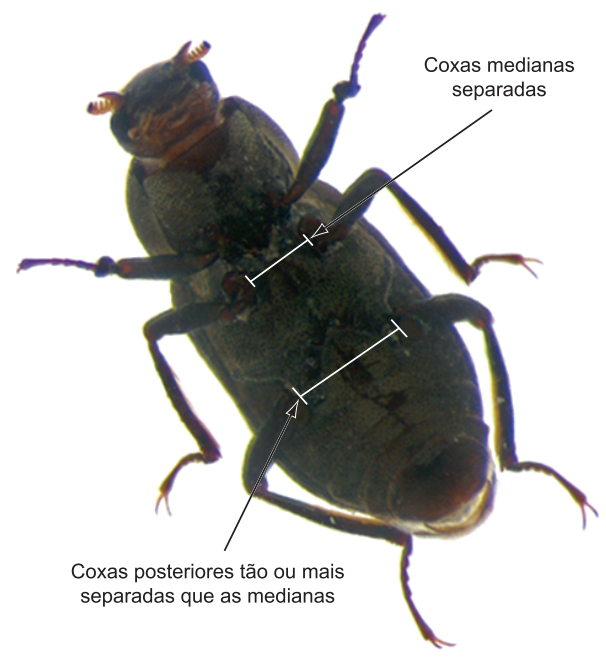

Figura 44. Dryopidae, adulto, vista ventral.

Figure 44. Dryopidae, adult, ventral view.

9. Antena clavada; palpo maxilar longo, geralmente maior ou igual ao comprimento da antena 10 (Figura 45)

9'. Antenas de outro tipo; palpo maxilar curto, menor que a antena 


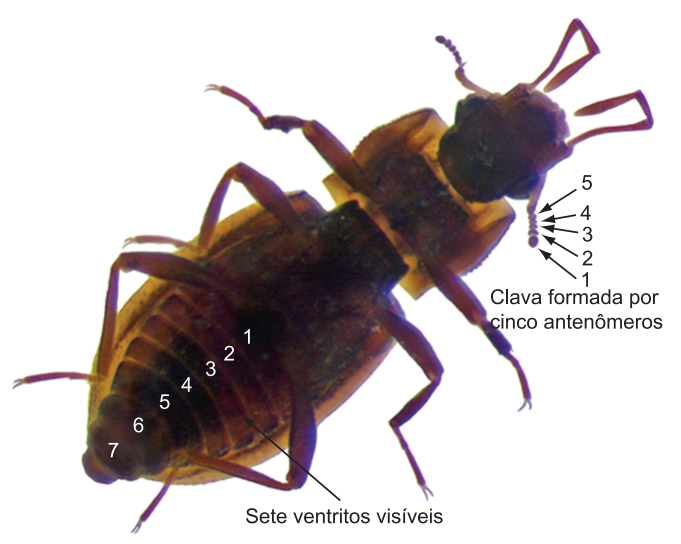

Figura 46. Hydraenidae, adulto, vista ventral.

Figure 46. Hydraenidae, adult, ventral view.

10'. Clava formada por 3 antenômeros, 5 ventritos visíveis.

Hydrophilidae (Figuras 47 e 48)

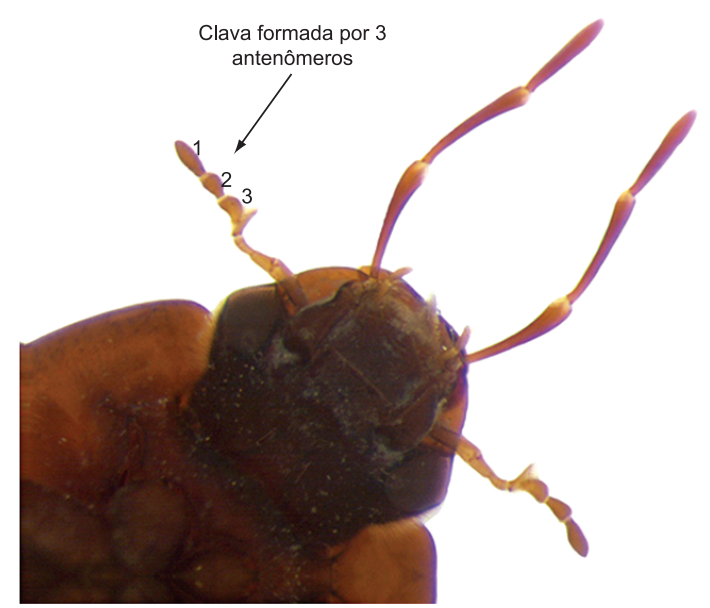

Figura 47. Hydrophilidae, adulto, vista ventral da cabeça e pronoto.

Figure 47. Hydrophilidae, adult, ventral view of the head and pronotum.

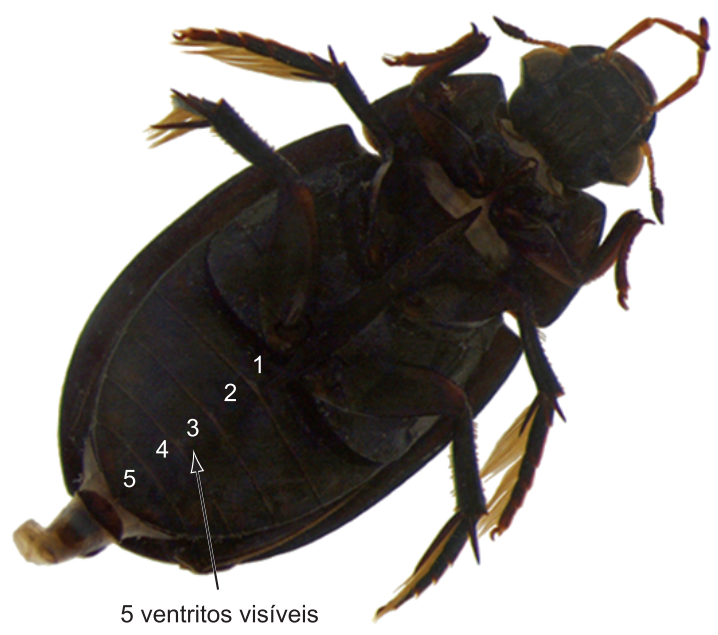

Figura 48. Hydrophilidae, adulto, vista ventral.

Figure 48. Hydrophilidae, adult, ventral view. 


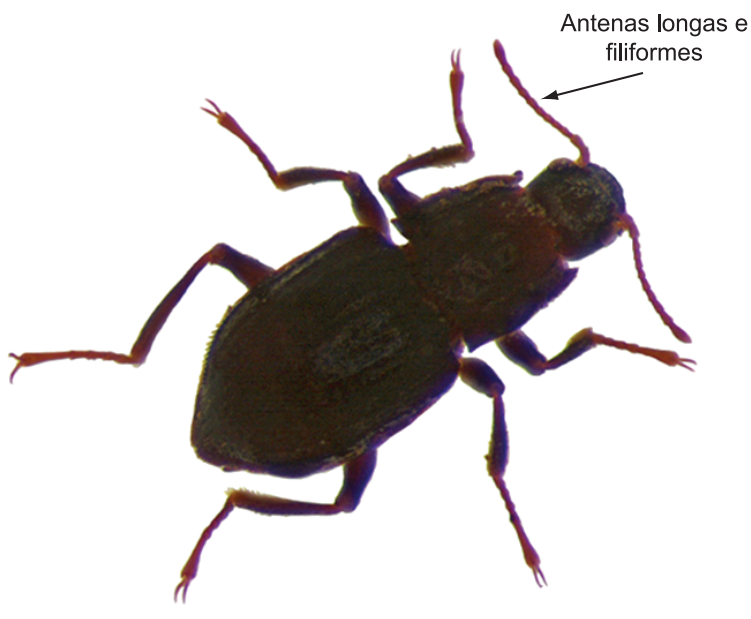

Figura 49. Elmidae, adulto, vista dorsal.

Figure 49. Elmidae, adult, dorsal view.

11'. Antenas curtas, últimos seis ou mais antenômeros pectinados

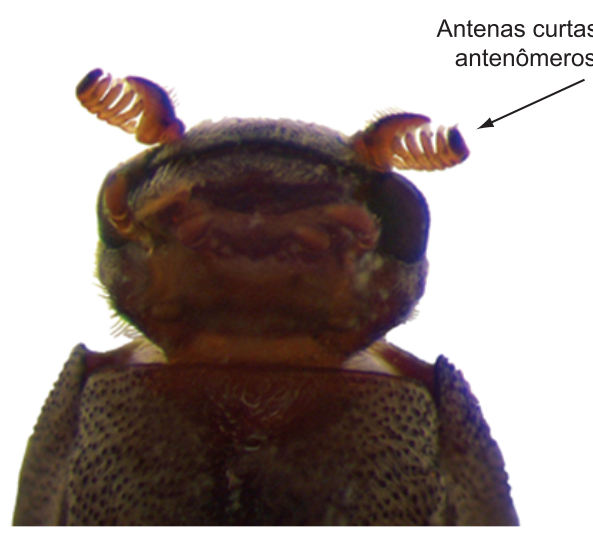

Figura 50. Dryopidae, adulto, vista ventral da cabeça.

Figure 50. Dryopidae, adult, ventral view of the head. 


\section{Agradecimentos}

Ao Prof. Dr. Claudio Gilberto Froehlich coordenador do Projeto BIOTA/FAPESP e aos colegas: Ana Emília Siegloch, Lívia Maria Fusari, Lucas Silveira Lecci, Márcia Regina Spies, Márcia Thaís Suriano, Fábio de Oliveira Roque e Tadeu Siqueira, pelos auxílios prestados em campo e exemplares gentilmente doados; aos alunos do Programa de Pós-graduação em Ecologia e Recursos Naturais (UFSCar), Daniel Gonçalves da Fonseca e Rogério Aparecido Libório, por testarem a chave proposta, e ao Dr. Mateus Pepinelli pelas sugestões e auxílio no tratamento das imagens. Este estudo foi financiado pela Fundação de Amparo à Pesquisa do Estado de São Paulo, dentro do Programa BIOTA/FAPESP e pelo Conselho Nacional de Desenvolvimento Científico e Tecnológico (CNPq).

\section{Referências Bibliográficas}

ARCHANGELSKY, M., MANZO, V., MICHAT, M.C. \& TORRES, P.L.M. 2009. Coleoptera. In Macroinvertebrados bentónicos sudamericanos. Sistemática y biologia (E. Domínguez \& H.R. Fernández, Ed.). Fundación Miguel Lillo, Tucumán, Argentina.
BENETTI, C.J., CUETO, J.A.R. \& FIORENTIN, G.L. 2003. Gêneros de Hydradephaga (Coleoptera: Dytiscidae, Gyrinidae, Haliplidae, Noteridae) citados para o Brasil, com chaves para identificação. Biota Neotrop. 3(1): http://www.biotaneotropica.org.br/v3n1/pt/abstract?identificationkey+BN00803012003 (último acesso em 17/06/2010).

BENETTI, C.J., FIORENTIN, G.L., CUETO, J.A.R. \& NEISS, U.G. 2006. Chave de identificação para famílias de coleópteros aquáticos ocorrente no Rio Grande do Sul, Brasil. Neotrop. Biol. and Conserv. 1(1):24-28.

BROWN, H.P. 1987. Biology of riffle beetles. Ann. Rev. Entomol. 32:253-273.

COSTA, C., VANIN, S.A. \& CASARI-CHEN, S.A. 1988. Larvas de Coleoptera do Brasil. Museu de Zoologia, Universidade de São Paulo, São Paulo, p.282.

CROWSON, R.A. 1981. The biology of Coleoptera. Academic Press, London.

JÄCH, M.A. \& BALKE, M. 2008. Global diversity of water beetles (Coleoptera) in freshwater. Hydrobiologia 595:419-442.

LARSON, D.J. 1985. Structure in temperate predaceous diving beetle communities (Coleoptera: Dytiscidae). Holarct. Ecol. 8:18-32.

LOPRETTO, E.C. \& TELL, G. 1995. Ecosistemas de aguas continentales: metodologías para su estudio. vol. 1, 2 e 3. Ediciones Sur, La Plata.

MUGNAI, R., NESSIMIAN, J.L. \& BAPTISTA, D.F. 2010. Manual de identificação de Macroinvertebrados aquáticos do Estado do Rio de Janeiro. Techinal Books Editora, Rio de Janeiro.

Recebido em 04/08/10 Versão reformulada recebida em 17/02/11

Publicado em 23/02/11 